ORIGINAL ARTICLE

\title{
Presence of vascular adventitial fibroblastic cells in diffuse- type gastric carcinomas
}

\author{
H Nakayama, H Enzan, E Miyazaki, N Kuroda, M Toi, M Hiroi, W Yasui
}

J Clin Pathol 2004;57:970-972. doi: 10.1136/jcp.2004.017137

See end of article for authors' affiliations .....................

Correspondence to: Dr H Nakayama, Department of Molecular Pathology, Graduate School of Biomedical Sciences, Hiroshima University, 1-2-3 Kasumi, Minami-ku, Hiroshima 734-8551, Japan; nakayamh@hiroshima-u. ac.jp

Accepted for publication 4 April 2003

\begin{abstract}
Aim: To investigate morphological changes in the tumour vessel adventitia, particularly the distribution of vascular adventitial fibroblastic cells (VAFCs)-namely, CD34 positive fibroblastic cells just outside the vascular media-in diffuse-type gastric carcinomas.

Method: In total , 18 surgically resected advanced typical diffuse-type gastric carcinomas and their normal tissues were examined. Immunostaining for CD34, CD31, high molecular weight caldesmon (HCD), and cytokeratin 8 (CAM5.2) was performed to detect VAFCs. VAFCs are positive for CD34 but negative for CD31, and are located just outside the vascular media (HCD positive vascular smooth muscle bundle). The areas just outside the vascular media in the whole maximum tumour cut surface were assessed, except the tumour growing edge, which was confirmed by immunostaining with CAM5.2. CD34 positive and CD31 negative cells just outside the vascular media were defined as VAFCs.

Results: VAFC containing vessels were seen in 17 of the 18 diffuse carcinoma tissues. Vessels lacking VAFCs were also detected in these 17 tumours. In contrast, all of the vessels lacked VAFCs in the remaining tumour. In the 18 samples of normal tissue, all of the vessels contained VAFCs.

Conclusions: These results suggest that the presence of VAFCs is associated with the infiltration of diffuse scattered gastric carcinoma cells.
\end{abstract}

n small vessels (except for capillaries), the vascular adventitia is a poorly defined layer composed of connective tissue and fibroblastic cells.

The vascular adventitia is a very important component from the point of view of vessel wall stabilisation. ${ }^{1}$ Vascular adventitial fibroblastic cells (VAFCs) change their phenotype ( $\alpha$ smooth muscle actin expressing fibroblastic cells, namely myofibroblasts) and migrate into the vascular media and intima in response to vascular injury. ${ }^{2}$ Transformed $\alpha$ smooth muscle actin expressing VAFCs-namely adventitial myofibroblasts-are associated with atherosclerosis of autologous aortocoronary saphenous vein grafts. ${ }^{3}$ Adventitial myofibroblasts also increase around and within saphenous veins after bypass grafting, and are closely associated with significant remodelling of vein grafts. ${ }^{4}$ Recently, it has been shown that hypoxic pulmonary artery adventitial fibroblasts secrete hypoxia inducible factors (HIF- $1 \alpha$, HIF- $2 \alpha$, and HIF$3 \alpha$ ) and trigger the proliferation of vascular smooth muscle cells. ${ }^{5}$ Hypoxic pulmonary artery adventitial fibroblasts themselves also differentiate into myofibroblasts. ${ }^{6}$

"The relation between vascular adventitial fibroblastic cells and diffuse scattered neoplastic cells has not yet been studied"

There have been only two reports regarding VAFCs in tumour pathology. ${ }^{78}$ No VAFCs are detected in the tumour stroma of intestinal-type and solid-type gastric carcinomas, which show gland formation and form large solid nests, respectively. $^{7}$ All vessels within subcutaneous vascular leiomyomas lack VAFCs. ${ }^{8}$ However, the relation between VAFCs and diffuse scattered neoplastic cells has not yet been studied.

In our present study, we investigated the morphological changes of the vascular adventitia, in particular the cellular components, in diffuse-type gastric carcinomas, by examining the distribution of VAFCs in diffuse-type gastric carcinomas and their normal tissues using CD34 as an immunohistochemical marker for VAFCs. ${ }^{7-9}$

\section{MATERIALS AND METHODS}

We examined 18 surgically resected advanced diffuse-type gastric carcinomas (five of the tumours invaded the muscularis propria without subserosal invasion and the remaining 13 tumours invaded the subserosa) and their normal tissues from the surgical pathology files of the department of pathology, Kochi Medical School, Japan, and its affiliated hospitals from 1994 to 2001. The definitions used for the histological classification were based on the criteria of Lauren $^{10}$ and our previous report. ${ }^{11}$ All 18 tumours were typical diffuse-type gastric carcinomas composed of scattered carcinoma cells and abundant fibrous and oedematous stroma. In general, typical diffuse-type early gastric carcinomas are very rare, and we were unable to examine these tumours in our present study.

The maximum tumour cut surface was immunostained in all 18 tumours; we used one to four paraffin wax blocks for each case.

Immunohistochemical studies were performed by the streptavidin-biotin method using the Histofine SAB-PO(M) kit (Nichirei, Tokyo, Japan). Four monoclonal antibodies against CD34 (clone MY10; Becton-Dickinson, Lexington, North Carolina, USA; 1/20 dilution; no pretreatment), CD31 (clone JC/70A; Dakopatts, Kyoto, Japan; 1/20 dilution; pronase pretreatment), high molecular weight caldesmon (HCD; clone h-CD; Dakopatts; 1/50 dilution; microwave pretreatment), and cytokeratin 8 (clone CAM5.2; BectonDickinson; 1/1 (prediluted); pronase pretreatment) were used. In addition to immunostaining for CD34, we also performed immunostaining for CD31 and HCD in all of the tumours and their normal tissues, to identify the vascular endothelial cells,

Abbreviations: $\mathrm{HCD}$, high molecular weight caldesmon; VAFC, vascular adventitial fibroblastic cell 


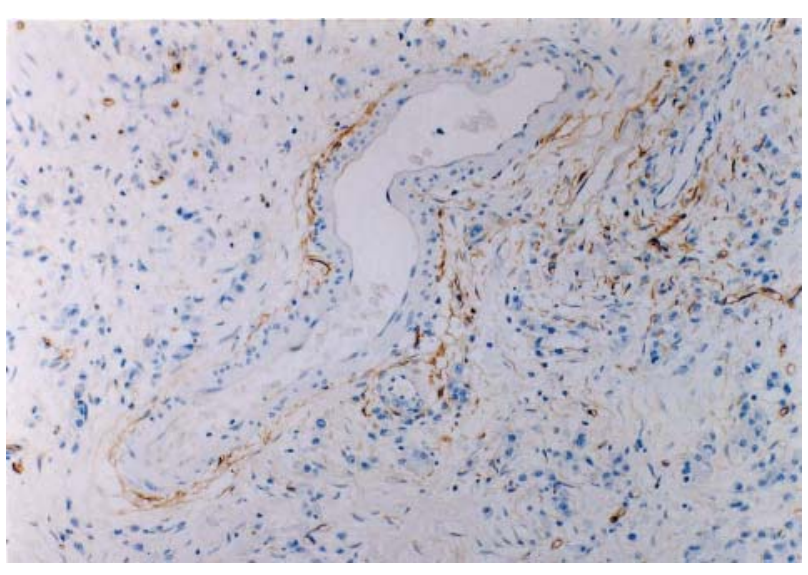

Figure 1 The presence of vascular adventitial fibroblastic cells in diffuse-type gastric carcinoma tissue. CD34 positive fibroblastic cells (vascular adventitial fibroblastic cells) are seen just outside the vascular media.

vascular media, and VAFCs in each specimen precisely ${ }^{72}{ }^{13}$ vascular endothelial cells are positive for CD34 and CD31; vascular smooth muscle cells are positive for HCD. We regarded CD34 positive and CD31 negative cells just outside the vascular media (HCD positive vascular smooth muscle bundle) to be VAFCs. As in our recent studies of gastric cancer, immunostaining for cytokeratin 8 was also performed to define the tumour growing edge in every specimen examined. ${ }^{11} 1415$

As we have already noted, ${ }^{7}$ it is impossible to discriminate tumour vessels from pre-existing normal vessels in tumour tissues of diffuse-type gastric carcinomas, so we assessed all vessels in the whole maximum tumour cut surface except the growing edge. The tumours were classified into two groups, namely: $(+)$, presence of VAFC containing vessels in diffusetype gastric carcinoma; and (-), absence of VAFC containing vessels in diffuse-type gastric carcinoma. Quantitative analysis was not performed.

\section{RESULTS}

Table 1 summarises the results.

VAFC (CD34 positive and CD31 negative stromal cells just outside the vascular media (HCD-positive vascular smooth muscle bundle)) containing vessels were detected in 17 of the 18 diffuse-type advanced gastric carcinoma tissues; these 17 diffuse-type carcinomas had VAFC containing vessels in the

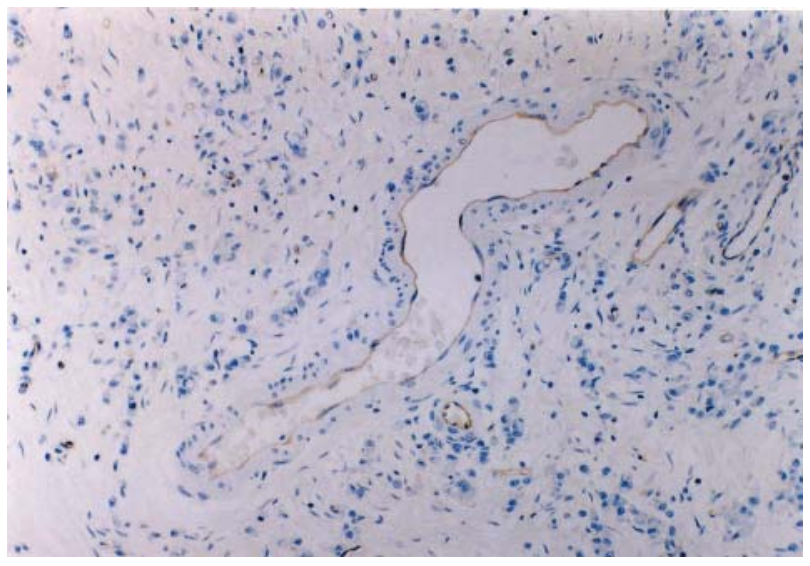

Figure 2 Diffuse-type gastric carcinoma tissue: staining for CD31 to distinguish CD34 positive stromal cells from vascular endothelial cells.
Table 1 Vascular adventitial fibroblastic cells (VAFCs) within the tumour tissue of 18 advanced diffuse-type gastric carcinomas

\begin{tabular}{llll}
\hline & & \multicolumn{2}{l}{ VAFC } \\
\cline { 3 - 4 } Depth of tumour invasion & Number of cases & $(+)$ & $(-)$ \\
\hline Muscularis propria & 5 & 4 & 1 \\
Subserosa & 13 & 13 & 0 \\
Total & 18 & 17 & 1 \\
\hline
\end{tabular}

$(+)$, presence of VAFC containing vessels within diffuse-type gastric carcinoma; $(-)$, absence of VAFC containing vessels within diffuse-type gastric carcinoma.

tumour tissues (figs 1-3) classified as $(+$ ). These 17 tumours also had vessels without VAFCs. No VAFC containing vessels were found in the remaining tumour, which was classified as $(-)$.

With regard to the depth of tumour invasion, four of the five diffuse carcinomas with invasion to the muscularis propria had VAFC containing vessels, and all of the 13 diffuse carcinomas with invasion to the subserosa had VAFC containing vessels.

All of the vessels in the 18 normal gastric tissues examined had VAFCs just outside the vascular media; all vessels contained VAFCs. The results regarding the normal gastric tissues agreed with our recent study. ${ }^{7}$

\section{DISCUSSION}

Recently, the role of the adventitia has received considerable attention in several pathological conditions of the cardiovascular system, including arteritis and atherosclerosis of coronary arteries and autologous saphenous vein grafts ${ }^{3}{ }^{4}$ 16; adventitial fibroblasts contribute to neointima formation and vascular remodelling. ${ }^{17}$ However, the role of the vascular adventitia in tumours has not been studied extensively-the only published studies being our two recent reports on the alteration of tumour vascular adventitia in intestinal-type and solid-type gastric carcinomas and subcutaneous vascular leiomyomas. ${ }^{78}$

It is impossible to differentiate tumour vessels from normal vessels involved in carcinomas. Therefore, we were unable to confirm whether the VAFC containing vessels within tumour tissues were tumour vessels, pre-existing normal vessels (involved within tumour tissues), or both. In our present study, we focused on the relation between VAFCs and diffuse-type gastric carcinomas. Diffuse-type gastric carcinomas have both VAFC containing vessels and VAFC lacking

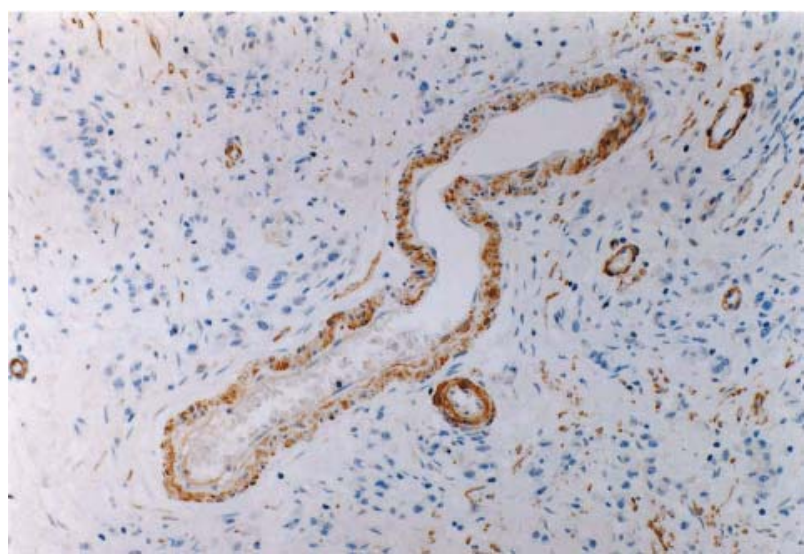

Figure 3 Diffuse-type gastric carcinoma tissue: staining for high molecular weight caldesmon to highlight the vascular media. 


\section{Take home messages}

- CD34 positive stromal cells just outside the vascular media (vascular adventitial fibroblastic cells; VAFCs) were found in advanced diffuse-type gastric carcinomas, whereas they are absent in the fumour stroma of intestinal-type and solid-type gastric carcinomas

- This suggests that the presence of VAFCs is associated with the infiltration of diffuse scattered gastric carcinoma cells

- Further molecular and biological investigations are needed to substantiate this hypothesis and to elucidate the pathobiological relevance of the presence of VAFCs in diffuse-type gastric carcinomas

vessels, whereas intestinal-type and solid-type gastric carcinomas have no VAFC containing vessels. ${ }^{7}$ These results suggest that the formation of both carcinomatous glands and solid tumour nests is associated with the lack of VAFCs, whereas diffuse scattered infiltrative growth of carcinoma cells is associated with the presence of VAFCs.

"Our results suggest that the formation of both carcinomatous glands and solid tumour nests is associated with the lack of vascular adventitial fibroblastic cells (VAFCs), whereas diffuse scattered infiltrative growth of carcinoma cells is associated with the presence of VAFCs"

On the basis of our recent paper, ${ }^{7}$ and our present results, we hypothesise that all VAFC containing vessels in diffusetype gastric carcinomas are pre-existing normal vessels, and that all VAFC lacking vessels in diffuse-type, intestinal-type, and solid-type gastric carcinomas are tumour vessels; in general, tumour vessels lack VAFCs. Accordingly, using the present hypothesis, 17 of the present 18 diffuse-type gastric carcinomas had VAFC containing vessels, namely preexisting normal vessels entrapped by tumour tissues.

In conclusion, CD34 positive stromal cells just outside the vascular media, namely VAFCs, were detected in advanced diffuse-type gastric carcinomas, suggesting that the presence of VAFCs is associated with the infiltration of diffuse scattered gastric carcinoma cells. To elucidate the pathobiological relevance of the presence of VAFCs in diffuse-type gastric carcinomas, and to substantiate this hypothesis, further molecular and biological investigations are needed.

\section{ACKNOWLEDGEMENTS}

The authors are grateful to Ms H Yamasaki, Ms M Yamamoto, Mr T Tokaji, and Mr Y Hayashi, Department of Pathology, Kochi Medical School, for their excellent technical assistance.

\section{Authors' affiliations}

H Nakayama, W Yasui, Department of Molecular Pathology, Graduate School of Biomedical Sciences, Hiroshima University, 1-2-3 Kasumi, Minami-ku, Hiroshima 734-8551, Japan

H Enzan, E Miyazaki, N Kuroda, M Toi, M Hiroi, Department of Pathology, Kochi Medical School, Kohasu, Okoh-cho, Nankoku, Kochi 783-8505, Japan

\section{REFERENCES}

1 Voung NV, Berry SC. The structure of vessels. In: Voung NV, Berry SC, eds. The pathology of vessels. Paris: Springer-Verlag, 2002:3-23.

2 Shi Y, Pieniek M, Fard A, et al. Adventitial remodeling after coronary arterial injury. Circulation 1996;93:340-8.

3 Shi Y, O'Brien JE, Mannion JD, et al. Remodeling of autologous saphenous vein grafts. The role of perivascular myofibroblasts. Circulation 1997;95:2684-93.

4 O'Brien JE, Shi Y, Fard A, et al. Wound healing around and within saphenous vein bypasss grafts. J Thorac Cardiovasc Surg 1997; 1 14:38-45.

5 Rose F, Grimminger F, Appel J, et al. Hypoxic pulmonary artery fibroblasts trigger proliferation of vascular smooth muscle cells: role of hypoxia-inducible transcription factors. FASEB J 2002;16:1660-1.

6 Short M, Nemenoff RA, Zawada WM, et al. Hypoxia induces differentiation of pulmonary artery adventitial fibroblasts into myofibroblasts. Am J Physiol Cell Physiol 2004;286:C416-25.

7 Nakayama H, Enzan H, Miyazaki E, et al. Lack of vascular adventitial fibroblastic cells in tumour stroma of intestinal-type and solid-type gastric carcinomas. J Clin Pathol 2004;57:183-5.

8 Nakayama $\mathrm{H}$, Enzan H, Miyazaki E, et al. Lack of CD34 positive stromal cells within angiomyomas (vascular leiomyomas). J Clin Pathol 2002;55:395-6.

9 van de Rijn M, Rouse RV. CD34. A review. Applied Immunohistochemistry 1994:2:71-80.

10 Lauren P. The two main types of gastric carcinoma. Diffuse and so-called intestinal type carcinomas. Acta Pathol Microbiol Scand 1965;64:31-49.

11 Nakayama H, Enzan H, Miyazaki E, et al. Alpha smooth muscle actin positive stromal cells in gastric carcinoma. J Clin Pathol 2002;55:741-4.

12 Miettinen $\mathbf{M}$, Lindenmayer AE, Chaubal A. Endothelial cell markers CD31, $\mathrm{CD} 34$, and $\mathrm{BNH} 9$ antibody to $\mathrm{H}$ - and $\mathrm{Y}$-antigens: evaluation of their specificity and sensitivity in the diagnosis of vascular tumours and comparison with von Willebrand factor. Mod Pathol 1994;7:82-90.

13 Miettinen MM, Sarlomo-Rikala M, Kovatich AJ, et al. Calponin and $\mathrm{h}$-caldesmon in soft tissue tumours: consistent $\mathrm{h}$-caldesmon immunoreactivity in gastrointestinal stromal tumours indicates traits of smooth muscle differentiation. Mod Pathol 1999;12:756-62.

14 Nakayama H, Enzan H, Miyazaki E, et al. Myofibroblasts at the tumour border of invasive gastric carcinomas: with special reference to histological type and tumour depth. Oncol Rep 2000;7:1011-15.

15 Nakayama $H$, Enzan $H$, Miyazaki E, et al. CD34 positive stromal cells in gastric adenocarcinomas. J Clin Pathol 2001:54:846-8.

16 Wilcox JN, Scott NA. Potential role of the adventitia in arteritis and atherosclerosis. Int J Cardiol 1996;54(suppl):S21-35.

17 Sartore S, Chiavegato A, Faggin E, et al. Contribution of adventitial fibroblasts to neointima formation and vascular remodeling. Circ Res 2001;89:1111-21. 